\title{
Chronic inflammation and cardiovascular risk
}

Ker JA, MBChB, MMed(Int), MD

Faculty of Health Sciences, University of Pretoria

Correspondence to: Prof James Ker, e-mail: james.ker@up.ac.za

SA Fam Pract 2010;52(1): 18

Much evidence points towards inflammation as a key regulatory process linking cardiovascular risk factors for atherosclerosis and its complications to an altered arterial biology. ${ }^{1}$ Systemic inflammatory conditions such as SLE, rheumatoid arthritis, Sjögren, systemic sclerosis, vasculitis, Crohn's disease and others are also associated with enhanced atherosclerosis.

There are many reports of the relationship between biomarkers of inflammation and cardiovascular risk. Generally, the clinical use of a biomarker depends on many factors (e.g. ease of measuring, cost, reproducibility etc). Thereare currently many such biomarkers of inflammation for diagnostic use such as myeloperoxidase, lipoprotein A2, interleukin -6 and C-reactive protein (CRP). CRP measured by a highly sensitive assay (hs CRP) has emerged as a leading biomarker of inflammation. Quite a number of largescale prospective cohort studies have shown that CRP predicts incident myocardial infarction, stroke and cardiovascular death. $\mathrm{CRP}$, along with parental history improves the prediction ability of the traditional Framingham risk score. There are strong indications that in people who fall in the intermediate risk category according to the Framingham risk score, an elevated CRP will push them into a higher risk category. Although there is strong evidence of association between CRP and cardiovascular risk, there is doubt whether CRP actually is causally linked to atherosclerosis. The question arises - should CRP be used to monitor therapy in a way that would improve clinical effectiveness of therapy? There is some evidence to show that if CRP, together with LDLcholesterol are lowered, the effectiveness will increase. In the Prove IT-Timi 22 trial, clinical outcomes were best among statin treated patients who not only achieved LDL-cholesterol levels below $1.8 \mathrm{mmol} / \mathrm{L}$ but who also achieved CRP levels below $2 \mathrm{mg} / \mathrm{L}^{2}$

The Jupiter trial tested this hypothesis prospectively. Maximum treatment benefit occurred with reduction of both LDL-cholesterol and CRP levels. ${ }^{3}$ Is there a future of targeting inflammation in atherosclerosis beyond statins? Systemic anti-inflammatory therapies such as corticosteroids and non-steroidal antiflammatory drugs are not suitable in atherosclerosis; in fact, they may increase thrombotic cardiovascular events.
There are indications that immuno-modulatory drugs, so called biologicals e.g. Blockade of Tumour necrosis factor-alpha may improve endothelial function and thus reduce the atherosclerotic risk in rheumatoid arthritis.

Inhibitors of lipoprotein associated phospholipase A2 are being tested. Vaccination with lipoprotein peptides is also being considered. Advances in our understanding of inflammation are being made rapidly and hopefully future therapeutic modalities will evolve.

\section{References}

1. Liby P, Ridker PM, Hansson G.K. Inflammation in atherosclerosis, J Am Coll Cardiol 2009:54:2129-2138.

2. Ridker PM, Morrow DA, et al. Relative efficacy of Atorvastatin $80 \mathrm{mg}$ and Pravastatin $40 \mathrm{mg}$ in achieving the dual goals of $\mathrm{LDL}<70 \mathrm{mg} / 2 \mathrm{~L}$ and CRP <2mg/L: an analysis of the Proveit Timi-22 trial. J Am Coll Cardiol 2005;45:1644-1648.

3. Ridker PM, Danielson E, et al. Rosuvastatin to prevent vascular events in men and women with elevated C-reactive protein $\mathrm{N}$ Eng $\mathrm{J}$ Med 2008;359:2195-2207.

4. Van Leuven SI, Franssen $\mathrm{R}$, et al. Review: Systemic inflammation as a risk factor for atherosclerosis. Rheumatology 2008;47:3-7. 